\title{
Feasibility Study of Industrial Applications of Micromegas Detectors
}

\author{
M. Schott and T. Alexopoulos
}

\begin{abstract}
The Micromegas (Micro-MEsh Gaseous Structure) detectors have been invented for the detection of ionizing particles in experimental physics, in particular in particle and nuclear physics. This detector technology is also one of the most promising candidates to be used during the upgrade of the particle detectors installed at the Large Hadron Collider. Current Micromegas detectors reach a spatial resolution of less than $50 \mu \mathrm{m}$. This would allow the usage of the Micromegas technology as an X-Ray imaging device with an active area of several $\mathrm{m} 2$ at very low production costs.

In this paper we present the latest status of Micromegas detector developments and discuss preliminary results on their photon detection performance in various energy-regimes and flux-rates. These performance tests are performed using a $8 \mathrm{keV}$ $X$-Ray source. We extrapolate our findings to energies and fluxes, which are relevant for possible industrial applications. It can be concluded that Micromegas detectors have a large potential to be used as active photon detection devices for a large variety of industrial applications for low energy, i.e. up to 50keV, X-Ray imaging.
\end{abstract}

Index Terms-Particle physics, medical physics, x-ray imaging, medical imaging, photon detection, particle detection.

\section{INTRODUCTION AND BASIC PRINCIPLES}

The Micromegas technique was first proposed in 1996 [1] and was under constant research, development and use from then on (e.g. [2]-[3]). The basic principle of a Micromegas detectors is illustrated in Fig. 1. A planar drift electrode is placed few $\mathrm{mm}$ above a readout electrode. The gap is filled with ionization gas, e.g. an 80:20 mixture of Argon and CO2. In addition, a metal mesh is placed $\sim 0.1 \mathrm{~mm}$ above the readout electrode. The region between drift electrode and mesh is called the drift region, while the region between mesh and readout electrodes is called the amplification region. Both the mesh and the drift electrode are set at negative high voltage, so that a electric field of $\sim 600 \mathrm{~V} / \mathrm{cm}$ is present in the drift region and a field of $\sim 50 \mathrm{kV} / \mathrm{cm}$ is present in the amplification region. The readout electrodes are set to ground potential.

Charged particles transversing the drift region ionize the gas. The resulting ionization electrons drift towards the mesh with a drift velocity of $5 \mathrm{~cm} / \mu \mathrm{s}$. The mesh itself appears transparent to the ionization electrons when the electric field in the amplification region is much larger than that in the drift region. Once reaching the amplification region, the ionization electrons cause a cascade of secondary electrons

Manuscript received August 10, 2011; revised September 30, 2012.

M. Schott is with the European Organisation for Nuclear Research (CERN), Switzerland (e-mail: mschott@cern.ch).

T. Alexopoulos is with National Technical Univ. of Athens Greece. (avalanche) leading to an amplification factor of $\sim 10^{4}$ in $2 \mathrm{~ns}$, which can be measured by the readout electrodes. Fig. 1 shows two incident particles, one entering the detection region perpendicular to the gap and one entering at $45^{\circ}$ degree. The two cases can be separated by the readout signal. While the perpendicular incident particle will typically cause a signal on only one readout strip , the second one will cause a time-dependent signal on several readout strips.

A significant step in the development of Micromegas detectors was achieved in 2006 and its known as bulk-Micromegas technology. This technology allows the industrial production of large scale Micromegas devices and is based on a printed board technology, which allows the precise placement of the metal mesh at an exact distance on top of the readout electronics. A detailed description can be found in [4].

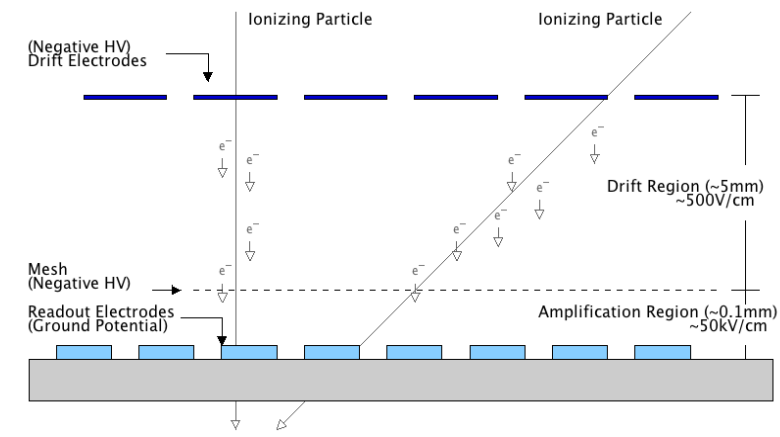

Fig. 1. Illustration of basic principle of a micromegas detector, with two incident ionizing particles. the incident particles ionize gas-atoms and the resulting secondary electrons drift to the lower mesh. once passing the lower mesh, the electrons cause a secondary electron cascade, leading to a signal amplification factor of several thousand.

The production costs per $\mathrm{m}^{2}$ of active detection area with a spatial resolution of $\sim 50 \mu \mathrm{m}$ are currently estimate to be below $\$ 10 \mathrm{k}$, not including readout electronics. This low cost is highly attractive for a large variety of scientific and industrial applications.

The paper is structured as follows. In Section 2, we briefly describe the current state of the art of the Micromegas research and development activities. This is followed by an assessment of the current photon detection capabilities of Micromegas detectors in Section 3. The requirements for commercial X-Ray detectors are discussed on Section 4, and the resulting conclusions are summarized.

\section{LATEST MicROMEGAS DEVELOPMENTS}

The thin amplification region together with its high electric field implies a large risk of sparking. Sparks can cause damage to the detector itself, on the underlying electronics, but lead also to significant dead-times. This serious 
disadvantage was overcome recently, with the development of spark resistant Micromegas chambers [5]. In this design, the readout strips are covered by a resistive protection layer . The protection is realized by a thin layer of insulator, with resistive paste $(\sim \mathrm{M} \Omega / \mathrm{cm})$ on top. It uses the same geometrical structure as the readout strips (see Fig. 2). This non-uniformity is aimed to reduce charge spread over seveal readout strips. The signal on the readout strips is then capacitively coupled to resistive strips. It has been shown that this design provides a spark-resistant layout for Micromegas chambers even in very high flux environments [6].

The basic Micromegas design can be easily extended to a two-dimensional readout, as shown in Fig. 2. The readout strips in the $\mathrm{x}$-direction are placed parallel to the resistive strips, while the readout-strips in the y-direction are placed perpendicular. All strips are separated by isolation material. The signal on the readout strips is again capacitively coupled to resistive strips. Hence it is expected that the induced signal on the $\mathrm{x}$-strips is smaller then the signal on the y-readout strips due to the larger distance to the resistive strips and screening effects. In order to ensure that the induced charge in both layers is of similar magnitude the lower readout-strips should be wider.

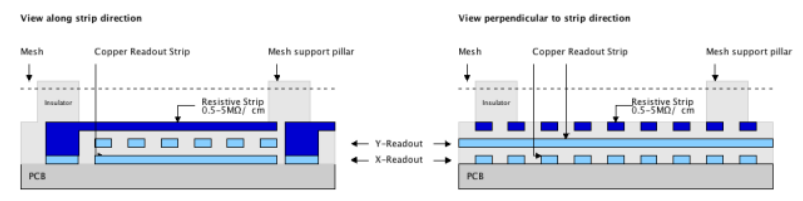

Fig. 2. Illustration of a spark protected micromegas detector with two-dimensional layout. view along the readout strips in $\mathrm{x}$ - (left) and $y$-direction (right). the resistive strips cover only the upper readout-layer, while the second readout0layer is separate with isolating material. This layer ensures a stable spark-protection.

\section{X-Ray Photon Detection Performance}

For testing two-dimensional, resistive strip Micromegas chambers under high photon fluxes, similar to those in industrial applications, an $8 \mathrm{keV} \mathrm{X-Ray} \mathrm{source} \mathrm{from} \mathrm{a} \mathrm{Cu}$ target was used. X-Ray rates up to $10 \mathrm{MHz} / \mathrm{cm} 2$ can be studied with this X-Ray source.

The test-chamber that was used for these studies was designed by the MAMMA collaboration (Muon ATLAS Micromegas Activity) [5], which focuses on the development and testing of large-area muon detectors for the upgrade program of the ATLAS detector at CERN. The chamber comprises 360 readout strips in both the $\mathrm{x}^{-}$and the $\mathrm{y}$-direction. The $\mathrm{x}$ - and $\mathrm{y}$-strips have a width of $150 \mu \mathrm{m}$ and are separated in equal distances by $250 \mu \mathrm{m}$. The amplification mesh covers an area of $10 \times 10 \mathrm{~cm}^{2}$ and is placed $128 \mu \mathrm{m}$ above the resistive strips. The amplification mesh (woven stainless steel, 157 lines/cm, line-diameter $18 \mu \mathrm{m}$ ) is fixed by so-called support pillars of $400 \mu \mathrm{m}$ diameter, which are made of the same material that is used for the insulation layer. The pillars are placed in a regular matrix with $2.5 \mathrm{~mm}$ distance in the $\mathrm{x}$ - and $\mathrm{y}$-direction. The drift-electrode is realized by a stainless steel mesh (138 wires/cm, line-diameter $22 \mu \mathrm{m}$ ), which is placed $5 \mathrm{~mm}$ above the amplification mesh.
An 85/15 gas-mixture of Argon and $\mathrm{CO}_{2}$ was used. The amplification and drift mesh were set to $830 \mathrm{~V}$ and $530 \mathrm{~V}$, respectively. The resulting gain factor per ionization electron is $3 \times 10^{3}$. A typical single photon event display is shown in Fig. 3. The incident photon causes a recorded charge distribution over five strips. The maximal recorded charge is reached after $\sim 100 \mathrm{~ns}$. The signal on the readout strips is capacitively coupled to resistive strips. As previously discussed, the induced signal on the $\mathrm{x}$-strips is smaller than the signal on the y-readout strips, due to the larger distance to the resistive strips and screening effects.

The photon interaction position can be estimated by the maximum of the recorded charge distribution, when assuming a perpendicular incidence. The maximum can be estimated by fitting a convoluted Landau-function with a Gaussian to the time-slice that contains the largest recorded charge. This leads to a measured spatial resolution of 30-60 $\mu \mathrm{m}$.
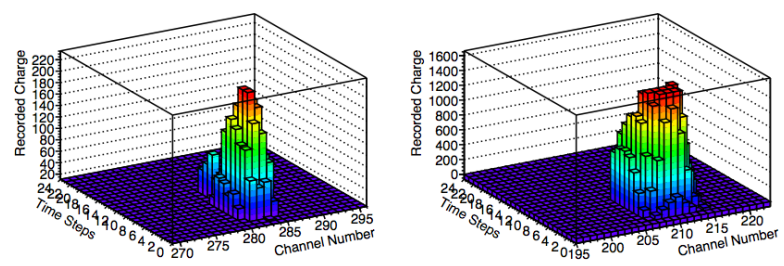

Fig. 3. Single photon signal in two-dimensional readout. the time-dependent measured charge on the in the x-readout (left) and the y-readout (right) is shown. fitting both distributions allows a precise position and time-measurement.

One time-step in Fig. 3 corresponds to $25 \mathrm{~ns}$, leading to an overall maximum signal-duration of $\sim 250 \mathrm{~ns}$ per incident photon. An intrinsic timing resolution of $5 \mathrm{~ns}$ can be reached, currently limited to $25 \mathrm{~ns}$ by the readout-electronics. In addition, it has been shown that the gain-factor reduces by no more than $20 \%$ at an incident photon rate of $107 \mathrm{~Hz} / \mathrm{cm} 2$.

The performance of the two-dimensional readout can be easily tested, by counting the recorded photon-rates over the full chamber. The photon interactions produce very localised signals, and therefore the rate of reconstructed events is reduced, if the interaction happens close to a support pillar. As a example, the recorded two-dimensional hit-distribution for the test-chamber with two-dimensional readout is shown in Fig. 4. The pillar support structures are clearly seen as regions with lower recorded rates. These tests are valid for soft X-Ray sources with photon energies between 1 and 10 $\mathrm{keV}$, but already illustrate the possibility to detect and record photons at highest rates with a good spatial resolution.

In order to extrapolate the results of these preliminary studies to higher photon energies, we use calculations based on the photon-gas interaction cross-sections. For $10 \mathrm{keV}$ photon energies, interaction cross-sections of $5.7 \times 1024 \mathrm{~b}$ and $1.1 \times 1023 \mathrm{~b}$ in the drift-region are expected, respectively, leading to 0.1-6 interactions on average within the drift region using an $\mathrm{Ar} / \mathrm{CO} 2$ gas mixture. The photon interaction cross-section is strongly energy dependent and proportional to $\mathrm{Z} 5 / \mathrm{E} \gamma$, where $\mathrm{Z}$ is the atomic number of the interaction material. Hence using Xe-gas within the drift-region leads to an expected cross-section of $1.5 \times 1023 \mathrm{~b}$ for $50 \mathrm{keV}$ photons, producing again to 0.1 average interactions within the drift region. 


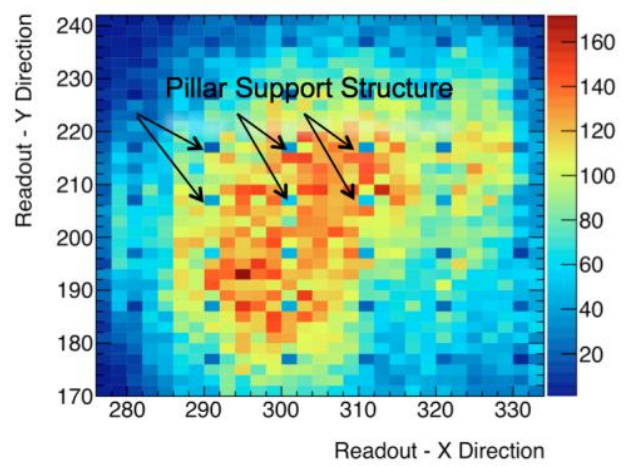

Fig. 4. X-ray counting rate of a two-dimensional micromegas chamber. regions of low measured intensity are shown blue, while high-active regions are marked red. it becomes clear, that regions close to the pillar support structure have a significant lower photon detection probability.

Hence a significant photon detection probability, at an incoming photon flux of $10^{7} \mathrm{~Hz} / \mathrm{cm}^{2}$, with a special resolution of $50-100 \mu \mathrm{m}$, and at photon energies between 1 and $50 \mathrm{keV}$ could be achieved with Micromegas detectors.

\section{CONCLUSION}

Large scale photon imaging devices with an active area of several $\mathrm{m}^{2}$ could have many potential applications, ranging from medical X-Ray imaging, to security imaging of objects. The expected X-Ray photon energies for these applications cover a range of $5 \mathrm{keV}$ to $100 \mathrm{keV}$, with an X-Ray photon rate of $10^{5} \mathrm{~Hz} / \mathrm{cm} 2$ to $10^{11} \mathrm{~Hz} / \mathrm{cm}^{2}$. The latter photon rate is expected for security scanning for container ships. For X-Ray imaging, the detector is required to have a dynamic range of about 104; that is, the ratio of the signal detected in air to the signal detected through the thickest part of the image is 10,000. Assuming a minimal rate of 10 detected photons per square unit $\left(100 \mu \mathrm{m}^{2}\right)$ per second leads to a photon-flux measurement capabilities of $10^{7} \mathrm{~Hz} / \mathrm{cm}^{2}$. These requirements exceed the detector performance expectations, discussed in [7]. Comparing these numbers with the presented performance measurements in section 4 , it can be concluded that Micromegas detectors have a large potential to be used as active photon detection devices for a large variety of industrial applications for low energy X-Ray imaging.

\section{REFERENCES}

[1] I. Giomataris et al., "Micro-pattern gaseous detectors," Nucl. Instrum. Methods vol. A376, no. 29, 1996

[2] T. Dafni et al., "New Micromegas for axion searches in CAST\}," Nucl. Instrum. Meth., vol. A628, PP. 172-176, 2011.

[3] D. Thers et al., "Micromegas as a large microstrip detector for the COMPASS experiment," Nucl. Instrum. Meth., vol. A469, PP. 133-146, 2001.

[4] I. Giomataris et al., "Micromegas in a bulk," Nucl Instrum. Methods, vol. A560, PP, 405, 2006.

[5] T. Alexopoulos et al., "A spark-resistant bulk-Micromegas chamber for high-rate applications," Nucl. Instrum. Meth., vol. A640, PP. 110-118, 2011

[6] T. Alexopoulos et al., "Development of large size Micromegas detector for the upgrade of the ATLAS muon system," Nucl. Instrum. Meth., vol. A617, PP. 161-165, 2010

[7] G. K. Fanourakis et al., "The use of the Micromegas technology for a new imaging system," Nucl Instrum. Methods, vol. A527, PP. 62-67, 2004 